\title{
Intermittent Hypoxia-Induced Carotid Body Chemosensory Potentiation and Hypertension Are Critically Dependent on Peroxynitrite Formation
}

\author{
Esteban A. Moya, ${ }^{1}$ Paulina Arias, ${ }^{1}$ Carlos Varela, ${ }^{1}$ María P. Oyarce, ${ }^{1}$ \\ Rodrigo Del Rio, ${ }^{2,3}$ and Rodrigo Iturriaga ${ }^{1}$ \\ ${ }^{1}$ Laboratorio de Neurobiología, Departamento de Fisiología, Facultad de Ciencias Biológicas, Pontificia Universidad Católica de Chile, \\ 8330025 Santiago, Chile \\ ${ }^{2}$ Centro de Investigación Biomédica, Universidad Autónoma de Chile, 8900000 Santiago, Chile \\ ${ }^{3}$ Dirección de Investigación, Universidad Científica del Sur, Lima, Peru
}

Correspondence should be addressed to Rodrigo Iturriaga; riturriaga@bio.puc.cl

Received 12 August 2015; Revised 22 September 2015; Accepted 27 September 2015

Academic Editor: Hanjun Wang

Copyright (C) 2016 Esteban A. Moya et al. This is an open access article distributed under the Creative Commons Attribution License, which permits unrestricted use, distribution, and reproduction in any medium, provided the original work is properly cited.

Oxidative stress is involved in the development of carotid body (CB) chemosensory potentiation and systemic hypertension induced by chronic intermittent hypoxia $(\mathrm{CIH})$, the main feature of obstructive sleep apnea. We tested whether peroxynitrite $\left(\mathrm{ONOO}^{-}\right)$, a highly reactive nitrogen species, is involved in the enhanced $\mathrm{CB}$ oxygen chemosensitivity and the hypertension during $\mathrm{CIH}$. Accordingly, we studied effects of Ebselen, an $\mathrm{ONOO}^{-}$scavenger, on 3-nitrotyrosine immunoreactivity (3-NT-ir) in the CB, the CB chemosensory discharge, and arterial blood pressure (BP) in rats exposed to $\mathrm{CIH}$. Male Sprague-Dawley rats were exposed to $\mathrm{CIH}$ $\left(5 \% \mathrm{O}_{2}, 12\right.$ times/h, $8 \mathrm{~h} /$ day) for 7 days. Ebselen $(10 \mathrm{mg} / \mathrm{kg} /$ day $)$ was administrated using osmotic minipumps and BP measured with radiotelemetry. Compared to the sham animals, $\mathrm{CIH}$-treated rats showed increased 3-NT-ir within the CB, enhanced CB chemosensory responses to hypoxia, increased BP response to acute hypoxia, and hypertension. Rats treated with Ebselen and exposed to CIH displayed a significant reduction in 3-NT-ir levels ( $60.8 \pm 14.9$ versus $22.9 \pm 4.2$ a.u.), reduced CB chemosensory response to $5 \% \mathrm{O}_{2}(266.5 \pm 13.4$ versus $168.6 \pm 16.8 \mathrm{~Hz})$, and decreased mean $\mathrm{BP}(116.9 \pm 13.2$ versus $82.1 \pm 5.1 \mathrm{mmHg})$. Our results suggest that $\mathrm{CIH}$-induced $\mathrm{CB}$ chemosensory potentiation and hypertension are critically dependent on $\mathrm{ONOO}^{-}$formation.

\section{Introduction}

The obstructive sleep apnea (OSA) syndrome, a worldwide sleep-breathing disorder, is recognized as an independent risk factor for hypertension [1-3]. OSA is characterized by repeated episodes of complete or partial obstruction of the upper airway during sleep, resulting in chronic intermittent hypoxic and hypercapnic events. Among the disturbances produced by OSA, chronic intermittent hypoxia $(\mathrm{CIH})$ is considered the main factor for the development of systemic hypertension [1-4]. Although the link between OSA and hypertension is well known, the mechanisms responsible for the hypertension are not entirely understood. OSA elicits oxidative stress, inflammation, and sympathoexcitation, which contribute to the endothelial dysfunction and hypertension $[1,2,5-8]$. Recently, it has been proposed that the carotid body (CB), the main $\mathrm{O}_{2}$ chemoreceptor, plays a pivotal role in the development of the enhanced sympathetic activity and the generation of the hypertension following $\mathrm{CIH}[1,2,7$, 8]. Indeed, CIH selectively enhanced CB chemosensory discharges during normoxia and hypoxia [8-12], which in turn led to a sustained potentiation of the sympathetic discharges to blood vessels, eliciting neurogenic hypertension $[1,2,7,12-$ 14]. The repetitive episodes of hypoxia-reoxygenation during $\mathrm{CIH}$ produce oxidative stress due to the accumulation of reactive oxygen species (ROS) [1-7]. The evidence indicates that the $\mathrm{CB}$ chemosensory potentiation induced by $\mathrm{CIH}$ is mediated by oxidative stress, which increases the levels of $\mathrm{CB}$ 
excitatory modulators such as angiotensin II and endothelin1 , and reduces the bioavailability of the inhibitory chemosensory modulator nitric oxide (NO) in the CB $[15,16]$. Peng et al. [8] proposed that the superoxide radical $\left(\mathrm{O}_{2}{ }^{-}\right)$participates in the potentiation of the rat $\mathrm{CB}$ chemosensory responses to hypoxia since they found that pretreatment of rats for 10 days before and concomitant with the exposure to $\mathrm{CIH}$ with manganese (III) tetrakis (1-methyl-4-pyridyl) porphyrin pentachloride (MnTMPyP), a superoxide dismutase (SOD) mimetic, prevented the $\mathrm{CB}$ chemosensory potentiation. We tested the hypothesis that oxidative stress contributes to the $\mathrm{CB}$ chemosensory potentiation and the progression of the hypertension in rats exposed to $\mathrm{CIH}$ [9]. We found that ascorbic acid treatment prevented systemic and local CB oxidative stress, the potentiation of $\mathrm{CB}$ chemosensory responses to hypoxia, and the hypertension in rats exposed to $\mathrm{CIH}$ for 21 days [9]. Although these results suggest that CB chemosensory potentiation is mediated by oxidative stress, it is matter of debate whether superoxide per se may increase the $\mathrm{CB}$ chemosensory discharges [17]. Thus, it is plausible that molecules downstream ROS formation may mediate the effects of oxidative stress on $\mathrm{CB}$ chemoreception. It is well known that $\mathrm{O}_{2}{ }^{-}$radical reacts with $\mathrm{NO}$ to produce peroxynitrite $\left(\mathrm{ONOO}^{-}\right)$, which can nitrate several proteins residues. Indeed, we have previously shown that $\mathrm{CIH}$ increases 3nitrotyrosine immunoreactivity (3-NT-ir) levels in the CB and that changes in 3-NT-ir correlate with the enhanced $\mathrm{CB}$ chemosensory responses to hypoxia following $\mathrm{CIH}$ [18]. Accordingly, it is plausible that nitrooxidative stress, through an $\mathrm{ONOO}^{-}$dependent pathway, may play a role in OSA pathophysiology. Thus, we studied if $\mathrm{ONOO}^{-}$is involved in the enhanced $\mathrm{CB}$ chemosensitivity and the generation and maintenance of the hypertension induced by $\mathrm{CIH}$. Therefore, we tested the effects of Ebselen treatment, a potent $\mathrm{ONOO}^{-}$ scavenger [19-21], on 3-NT-ir accumulation in the CBs, CB chemosensory responses to hypoxia, and arterial blood pressure (BP) in conscious rats exposed to $\mathrm{CIH}$, a well stablished experimental model of OSA $[7,9,15,18]$.

\section{Material and Methods}

2.1. Animals and Intermittent Hypoxia Protocol. Experiments were performed on adult male Sprague-Dawley rats weighting $200 \mathrm{~g}$ fed with standard diet ad libitum and kept on a 12:12hour light dark cycle. Room temperature was maintained between 23 and $25^{\circ} \mathrm{C}$. All the experimental procedures were approved by the Bioethical Committee of the Biological Sciences Faculty, Pontificia Universidad Católica de Chile, Santiago, Chile, and were performed according to the National Institutes of Health Guide (NIH, USA) for the care and use of animals. Unrestrained, freely moving rats were housed in individual chambers $(12 \mathrm{~cm} \times 35 \mathrm{~cm}, 3 \mathrm{~L})$ and exposed to hypoxic cycles of $5 \%$ inspired $\mathrm{O}_{2}$ for $20 \mathrm{~s}$, followed by $280 \mathrm{~s}$ of room air, 12 times per hour during 8 hours a day, or exposed to sham air-air cycles, emulating the same conditions of noise, temperature, and flow $[7,9,10]$. Rats were exposed to $\mathrm{CIH}$ from 8:00 AM to 16:00 PM. The $\mathrm{O}_{2}$ level inside the chambers was continuously monitored with an oxygen analyzer (Ohmeda 5120, BOC Healthcare, Manchester, UK) and the $\mathrm{CO}_{2}$ levels and humidity were maintained at low levels by continuous air extraction.

2.2. Carotid Body Chemosensory Recording. The CB chemosensory discharges were measured in situ as previously described $[9,10,12]$. Rats were anesthetized with sodium pentobarbitone $(40 \mathrm{mg} / \mathrm{kg})$ and additional doses were given to the animal when necessary to maintain a level of surgical anesthesia. Rats were placed on supine position and the body temperature was maintained at $38 \pm 0.5^{\circ} \mathrm{C}$ with a heating pad. The trachea was cannulated for gases administration. One carotid sinus nerve was dissected and placed on a pair of platinum electrodes and covered with mineral oil. The neural signal was preamplified (Grass P511, Grass Instruments, Quincy, MA, USA), filtered $(30 \mathrm{~Hz}-1 \mathrm{kHz})$, and fed to an electronic spike-amplitude discriminator, allowing the selection of action potentials of particular amplitude above the noise to be counted with a frequency meter, thus being able to measure the frequency of carotid chemosensory discharges $\left(f_{x}\right)$ expressed in $\mathrm{Hz}$. The carotid sinus electroneurogram was continuously monitored using an oscilloscope. Baroreceptor activity, which consisted of repetitive and rhythmic discharges synchronized with the systolic arterial blood pressure, was eliminated by crushing the baroreceptor fibers between the carotid bifurcation and the CB. This procedure resulted in the complete elimination of discharge synchronized with arterial blood pressure. $100 \% \mathrm{O}_{2}$ (Dejours test) was used to confirm that all the neural activities recorded correspond to chemosensory discharge $[9,12]$. The contralateral carotid sinus nerve was cut to prevent vascular and ventilatory reflexes evoked by hypoxic activation. The CB chemosensory frequency of discharge $\left(f_{x}\right)$ was measured in response to several levels of inspired $\mathrm{PO}_{2}(\sim 5-670 \mathrm{mmHg}$, applied for 20-30 s), by averaging the maximal values during the semiplateau of the chemosensory response. Thus, $f_{x}$ is the absolute value of chemosensory rate of discharge. The $\mathrm{O}_{2}$ levels were measured with an oxygen analyzer (Ohmeda 5120, BOC Healthcare, Manchester, UK).

2.3. Arterial Blood Pressure Telemetry. In a subset of rats, arterial blood pressure (BP) was recorded using radiotelemetry. Briefly, rats were anesthetized with 5\% isoflurane and maintained with $1-2 \%$ isoflurane in $100 \% \mathrm{O}_{2}$ during the surgical procedure. An abdominal incision was performed to isolate the abdominal aorta. The tip of a cannula-coupled telemetry device (Telemetry Research TRM54P, Millar USA) was inserted into the abdominal aorta and fixed with methacrylate. After this procedure, the abdominal incision was sutured in layers. At the end of the surgery, rats received an i.p. injection of enrofloxacin (1\%) and ketoprofen (1\%) and were supplied during 3 subsequent days with the same dose of enrofloxacin in the drinking tap water. Physiological variables were acquired with an analogue-digital system (PowerLAB 8SP, ADInstruments, Australia) and analyzed with the Chart 7.2-Pro software. BP was measured after one week of recovery. For quantification of the baseline BP during normoxia, we averaged 10 minutes of $\mathrm{BP}$ signal, recorded 30 minutes before the beginning of the $\mathrm{CIH}$ protocol. To quantify $\triangle \mathrm{BP}$ response 
evoked by acute $5 \% \mathrm{O}_{2}$, we measured the difference between the maximal BP averaged for $5 \mathrm{~s}$ and the baseline BP values averaged for $1 \mathrm{~min}$ before the acute hypoxic challenge.

2.4. Experimental Procedure and Ebselen Administration. The peroxynitrite targeted antioxidant Ebselen (Enzo Life Sci, Inc., Farmingdale, NY, USA) was administrated with osmotic minipumps (2ML4, Alzet Scientific Products, Chevy Chase, MD, USA). Rats were anesthetized with isoflurane in $100 \%$ $\mathrm{O}_{2}$, and osmotic minipumps were implanted subcutaneously on the back. Pumps were filled with $33.3 \mathrm{mg}$ Ebselen in $1 \mathrm{~mL}$ of $80 \%$ DMSO in saline, to achieve a delivering rate of $10 \mathrm{mg} / \mathrm{kg} /$ day, a similar dose used in other studies [22, 23]. Control animals were implanted with osmotic minipumps filled with DMSO 80\% in saline solution. After surgical procedures, the rats were treated with enrofloxacin and ketoprofen as mentioned before. Due to the nature of the experiments related to the study of $\mathrm{CB}$ chemosensory activity, a crosssectional study was performed. Twenty-four rats were randomly divided into 3 groups: one control group, exposed to sham conditions and treated with Ebselen (Ebselen-Sham); a second group, exposed to $\mathrm{CIH}$ and treated with vehicle (Vehicle-CIH); and a third group, exposed to $\mathrm{CIH}$ and treated with Ebselen during the hypoxic protocol (Ebselen CIH). To study the therapeutic effect of Ebselen on the $\mathrm{CIH}$-induced hypertension, we performed a longitudinal study. Rats with indwelling catheter from telemetry devices were exposed first to sham conditions for 7 days and then to $\mathrm{CIH}$ for other 7 days. At the end of the $\mathrm{CIH} 7$-day exposure, an osmotic minipump filled with Ebselen was subcutaneously implanted and the rats were kept another week in $\mathrm{CIH}$.

2.5. Nitrotyrosine Immunohistochemistry. At the end of the $\mathrm{CB}$ chemosensory studies, anesthetized rats were perfused intracardially with saline at $\mathrm{pH} 7.4$ for $15 \mathrm{~min}$ followed by buffered $4 \%$ paraformaldehyde (PFA, Sigma, St. Louis, MO, USA). The carotid bifurcations were dissected and postfixed in the same fixative solution for $12 \mathrm{~h}$ to $4^{\circ} \mathrm{C}$. Then, the samples were dehydrated in ethanol, included in paraffin, cut in $5 \mu \mathrm{m}$ sections, and mounted on silanized slides. Samples were deparaffinized and exposed to antigen-retrieval solution (citrate buffer 1 M, pH 6.0) as previously described $[9,18]$. Samples were then incubated in $0.3 \% \mathrm{H}_{2} \mathrm{O}_{2}$ solution and blocked using normal horse serum (ABC, Vectastain kit, Vector) for 1 hour. The samples were then incubated with primary antibody against 3-NT (1:500, number A21285, Molecular Probes) overnight at $4^{\circ} \mathrm{C}$. The immunoreactivity staining was detected using a streptavidin-peroxidase kit (ABC, Vectastain kit, Vector) and revealed at $37^{\circ} \mathrm{C}$ in dark chamber with $3,3^{\prime}$ diaminobenzidine tetrahydrochloride (DAB, Sigma). Samples were counterstained with Harris haematoxylin and mounted with Entellan (Merck, Whitehouse station, NJ, USA). Photomicrographs were taken at 100x using a CCD camera coupled to an Olympus CX 31 microscope (Olympus Corp., USA), digitized, and analyzed using ImageJ software (NIH, Betheseda, MD, USA). We measure two nonconsecutive $\mathrm{CB}$ sections per rat, obtaining four CB photographs from each one of those sections. The positive 3-NT-ir, averaged
TABLE 1: Systolic, diastolic, and pulse pressure measured in normoxia in the same rats exposed to sham, $\mathrm{CIH}$, or Ebselen $\mathrm{CIH}$ condition.

\begin{tabular}{lccc}
\hline & Sham & CIH & Ebselen CIH \\
\hline$P_{s}(\mathrm{mmHg})$ & $109.7 \pm 2.7$ & $138.0 \pm 12.6^{*}$ & $110.0 \pm 3.5$ \\
$P_{d}(\mathrm{mmHg})$ & $79.1 \pm 2.7$ & $106.4 \pm 13.5^{*}$ & $71.2 \pm 4.9$ \\
$P_{p}(\mathrm{mmHg})$ & $30.6 \pm 1.4$ & $31.7 \pm 2.0$ & $32.9 \pm 3.0$ \\
\hline
\end{tabular}

$P_{s}$, systolic, $P_{d}$, diastolic, and $P_{p}$ pulse arterial pressure $\left(P_{s}-P_{d}\right) .{ }^{*} p<$ $0.05, \mathrm{CIH}$ versus sham and Ebselen CIH and Newman-Keuls after Repeated Measures ANOVA, $n=4$ rats.

TABLE 2: Arterial blood pressure responses to $5 \% \mathrm{O}_{2}$ measured during normoxia in the same rats exposed to sham, $\mathrm{CIH}$, or Ebselen $\mathrm{CIH}$ condition.

\begin{tabular}{lccc}
\hline & Sham & CIH & Ebselen CIH \\
\hline$\Delta P_{s}(\mathrm{mmHg})$ & $14.6 \pm 3.5$ & $45.5 \pm 4.6^{*}$ & $14.4 \pm 5.7$ \\
$\Delta P_{d}(\mathrm{mmHg})$ & $4.7 \pm 4.3$ & $19.7 \pm 4.3$ & $3.6 \pm 3.5$ \\
$\Delta P_{p}(\mathrm{mmHg})$ & $9.9 \pm 1.9$ & $17.2 \pm 10.3$ & $10.8 \pm 4.2$ \\
\hline
\end{tabular}

$\Delta P_{s}$, max-baseline systolic arterial pressure, $\Delta P_{d}$, max-baseline diastolic arterial pressure, and $\Delta P_{p}$, max-baseline pulse arterial pressure. ${ }^{*} p<0.05$, $\mathrm{CIH}$ versus sham and Ebselen $\mathrm{CIH}$ and Newman-Keuls after Repeated Measures ANOVA, $n=4$ rats.

from the eight CB fields, was expressed as optical integrated intensity, in arbitrary units.

2.6. Statistical Data Analysis. Data was expressed as mean \pm SEM. For cross-sectional studies (Figures 1 and 2), statistical analysis was performed using one-way or two-way ANOVA tests followed by Bonferroni post hoc analysis. For longitudinal studies (Figures 3 and 4 and Tables 1 and 2), Repeated Measures one-way ANOVA followed by Newman-Keuls post hoc comparisons was used. $P<0.05$ was set as the level of statistical significance for both studies.

\section{Results}

3.1. Effects of Ebselen on the CIH-Induced Increase of 3-NT-ir in the $C B$. The exposure to $\mathrm{CIH}$ for 7 days produced a marked increase in the 3-NT-ir levels in the CB (Figure 1(a)). Indeed, we found a 2.5 -fold increase in 3-NT-ir in the CB from CIHtreated rats compared to the levels observed in sham rats. The administration of the peroxynitrite scavenger Ebselen to the rats during the $\mathrm{CIH}$ exposure prevented the $\mathrm{CIH}$-induced increase of 3-NT-ir in the CB (Figure 1(b)). Figure 1(b) shows the quantification of the effects of Ebselen on the 3-NT-ir accumulation induced by $\mathrm{CIH}$. Rats exposed to $\mathrm{CIH}$ and treated with Ebselen showed $60 \%$ of reduction in 3-NT-ir as compared with the $\mathrm{CIH}$ rats treated with vehicle $(60.8 \pm 14.9$ versus $22.9 \pm 4.2$ a.u., $P<0.05$, and $\mathrm{CIH}$ and $\mathrm{CIH}$ Ebselen rats, resp.).

\subsection{Ebselen Prevented CB Chemosensory Potentiation Induced} by $\mathrm{CIH}$. To assess the effect of Ebselen on $\mathrm{CB}$ chemosensory activity, we measured the frequency of chemosensory discharge $\left(f_{x}\right)$, from the carotid sinus nerve from rats exposed to $\mathrm{CIH}$ and treated with Ebselen (Figure 2). The exposure to 

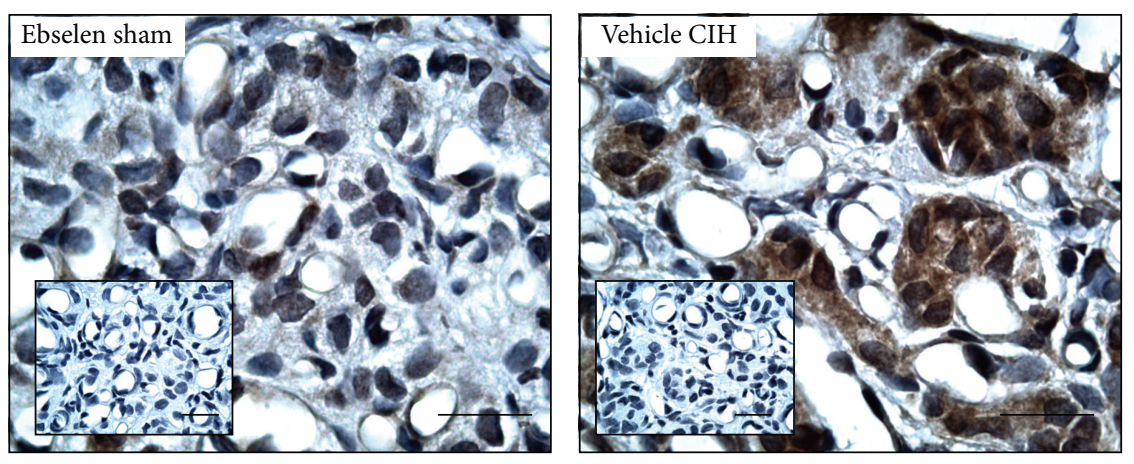

(a)

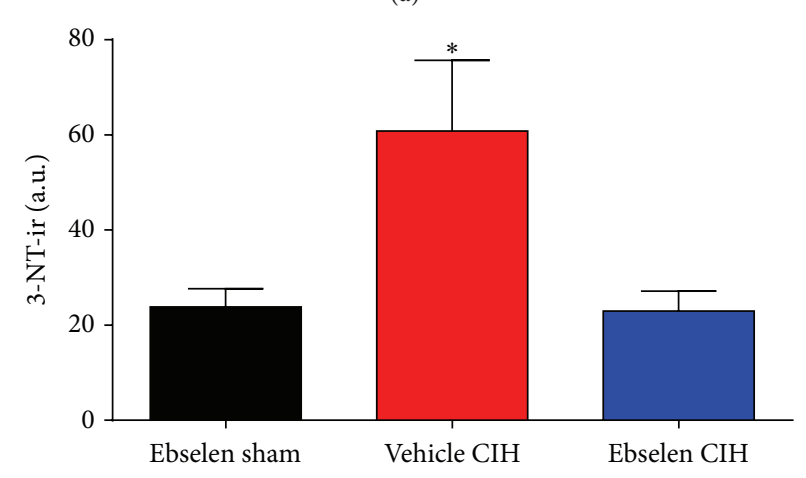

(b)

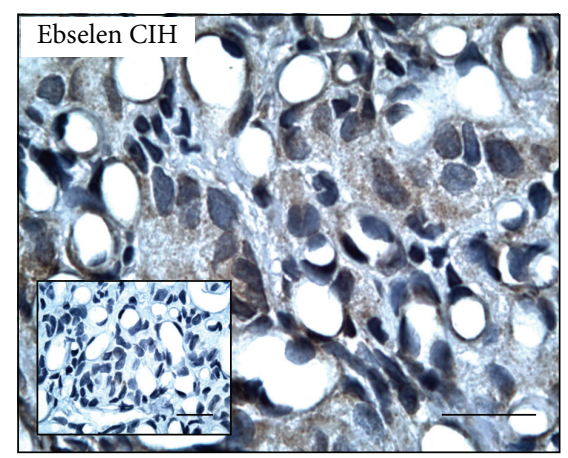
FIGURE 1: Ebselen treatment prevented the increased levels of 3-NT-ir. (a) Representative effects of Ebselen on positive 3-NT immunoreactivity
(3-NT-ir) in CBs from rats exposed to CIH. Inset, negative controls omitted inclusion of primary antibody. Scale bars $20 \mu \mathrm{m}$. (b) Summary of the effects of Ebselen on 3-NT-ir measured in CBs. ${ }^{*} P<0.05$, vehicle $\mathrm{CIH}$ versus Ebselen sham and Ebselen CIH, and Bonferroni after one-way ANOVA, $n=5$ rats per group.

CIH for 7 days increases the baseline CB chemosensory and the discharge evoked by hypoxia. Indeed, Ebselen treatment prevented the potentiation of the hypoxic $\mathrm{CB}$ chemosensory response in $\mathrm{CIH}$ rats (Figure 2). The two-way ANOVA analysis showed a significant increase of $\mathrm{CB}$ chemosensory discharge for different levels of inspired $\mathrm{PO}_{2}$ in rats exposed to $\mathrm{CIH}(P<0.01)$. The treatment with Ebselen during $\mathrm{CIH}$ exposure effectively prevented the $\mathrm{CB}$ increased responses to several levels of hypoxia (Figure 2(b)).

\subsection{Effects of Ebselen on the CIH-Induced Hypertension.} Exposure to 7 days of $\mathrm{CIH}$ produced a significant increase in baseline BP measured in normoxia (Figure 3). We found that, after one week of $\mathrm{CIH}$ exposure, the mean arterial blood pressure (MABP) increased about $25 \mathrm{mmHg}$ compared to the value measured during sham condition $(89.3 \pm 2.5 \mathrm{mmHg}$ versus $116.9 \pm 13.2 \mathrm{mmHg}, P<0.05$ sham versus $\mathrm{CIH}$, resp.). Remarkably, Ebselen treatment normalized MABP during $\mathrm{CIH}$ to similar levels to those observed during sham conditions $(82.1 \pm 5.1 \mathrm{mmHg}$, Figure 3$)$. The values for baseline systolic $\left(P_{s}\right)$, diastolic $\left(P_{d}\right)$, and pulse pressure $\left(P_{p}\right)$ are summarized in Table 1 . We did not find significant differences in resting heart rate (HR) between animals exposed to sham, $\mathrm{CIH}$, and Ebselen $\mathrm{CIH}$ conditions (sham $321.9 \pm 15.5, \mathrm{CIH}$ $383.6 \pm 20.3$, and CIH Ebselen $351.8 \pm 36.3$ beats per minute,
Figure 3(c), $P>0.05$, one-way ANOVA). In a separate experimental series, we measured MABP and HR in 3 sham rats after one week of the implantation of osmotic pumps containing the vehicle (DMSO 80\%). We did not find any differences (MABP $97.4 \pm 3.9 \mathrm{mmHg}$, HR $333.1 \pm 5.3$ beats per minute) related to the values recorded in rats implanted with pumps containing Ebselen in DMSO in sham conditions for one week.

In addition, we measured the BP response evoked by acute hypoxia (Figure 4). Acute hypoxic episodes $\left(5 \% \mathrm{O}_{2}\right)$ in sham rats produced a mild increase in $\mathrm{BP}(\triangle \mathrm{MABP}=8.0 \pm$ $3.9 \mathrm{mmHg}$ ). In contrast, after one week of $\mathrm{CIH}$ exposure, the $\mathrm{BP}$ response to the same level of hypoxia was largely increased $(\triangle \mathrm{MABP}=28.1 \pm 4.1 \mathrm{mmHg})$. Ebselen treatment during $\mathrm{CIH}$ exposure normalized $\mathrm{BP}$ responses to hypoxia $(\triangle \mathrm{MABP}=$ 7.2. $\pm 3.9 \mathrm{mmHg}$ ). The mean values for $\Delta P_{s}, \Delta P_{d}$, and $\Delta P_{p}$ during acute hypoxic stimulus are shown in Table 2. Therefore, treatment with Ebselen effectively restores the normal arterial pressure response to hypoxia, even in the presence of $\mathrm{CIH}$. The increases in BP following acute hypoxic stimulation produced a reflex bradycardia in all three conditions (sham, $\mathrm{CIH}$, and Ebselen $\mathrm{CIH}$ ). The $\Delta \mathrm{HR}$ response to hypoxia did not reach statistical significance between the treatments $(P>$ 0.05 , Sham $=21.8 \pm 28.8, \mathrm{CIH}=90.8 \pm 54.5$, and Ebselen $\mathrm{CIH}=$ $60.8 \pm 64.8$ beats per minute, Figure $4(\mathrm{c}), P<0.05$, one-way ANOVA). 

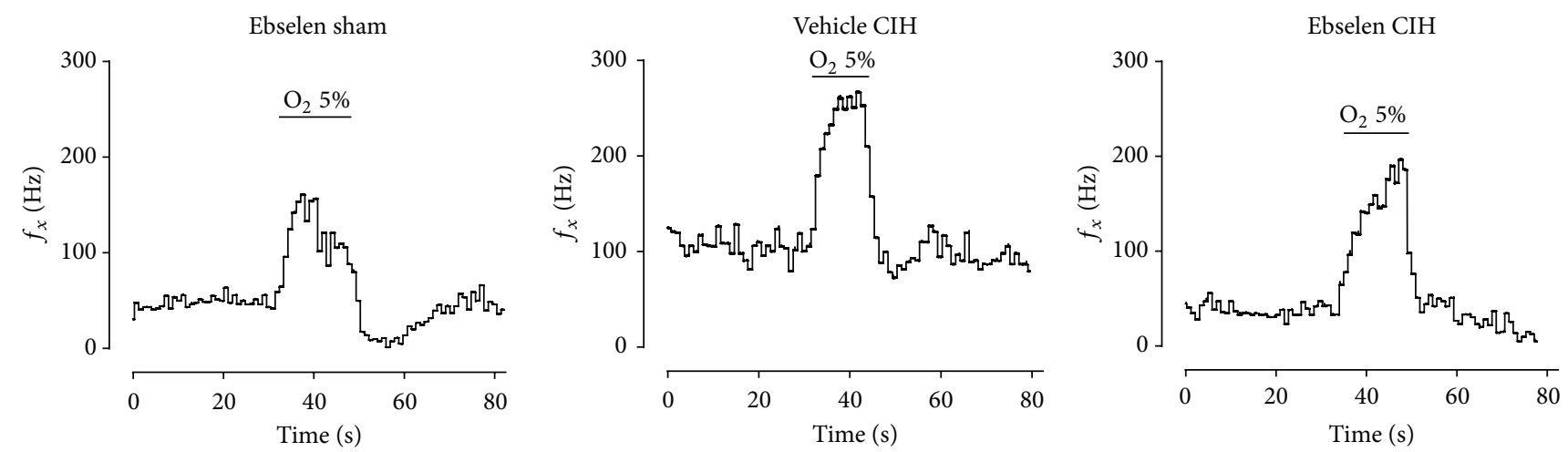

(a)

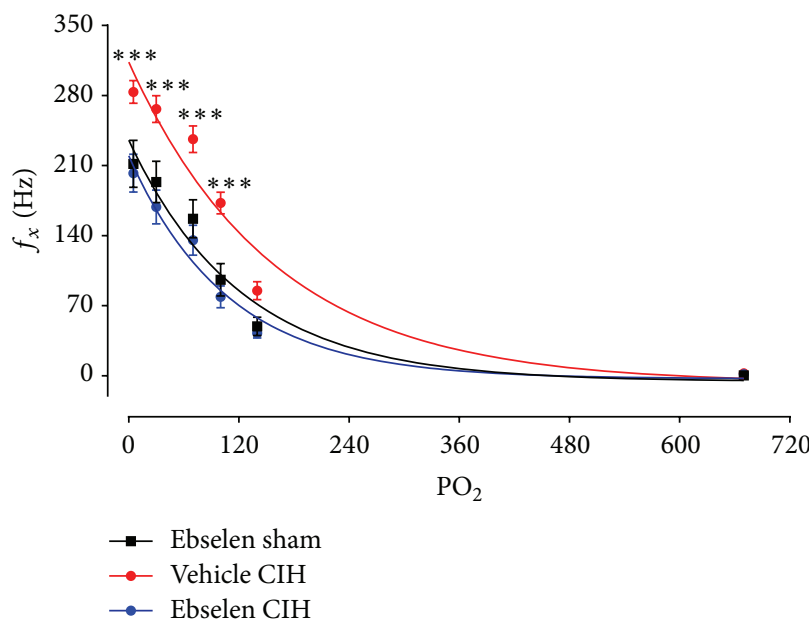

(b)

Figure 2: Ebselen treatment prevented the $\mathrm{CB}$ chemosensory potentiation in rats exposed to CIH. (a) Recordings of the CB frequency of chemosensory discharge $\left(f_{x}\right)$ measured from the carotid sinus nerve in response to acute hypoxia $\left(5 \% \mathrm{O}_{2}\right)$ in rats treated with Ebselen or vehicle and exposed to sham or $\mathrm{CIH}$ conditions. The enhanced $\mathrm{CB}$ chemosensory response induced by $\mathrm{CIH}$ (vehicle $\mathrm{CIH}$ ) was prevented by Ebselen (Ebselen CIH). (b) Summary of the effect of the Ebselen treatment at different levels of inspired $\mathrm{PO}_{2}(\mathrm{mmHg})$ on $f_{x}$. ${ }^{* * *} P<0.001$, vehicle $\mathrm{CIH}$ versus Ebselen sham and Ebselen $\mathrm{CIH}$, and Bonferroni after two-way ANOVA, $n=8$ rats per group.

\section{Discussion}

Present results show that Ebselen prevented the accumulation of 3-NT-ir in the CB and the enhanced CB chemosensory discharges induced by CIH. Indeed, Ebselen reduced the baseline chemosensory discharge and the responses to hypoxia (Figure 2), confirming observations showing that antioxidant treatment prevents the potentiation of the rat CB chemosensory response to hypoxia induced by $\mathrm{CIH}[8,9]$. Furthermore, we found that administration of Ebselen, once rats already developed hypertension induced by $\mathrm{CIH}$ exposure, was able to normalize baseline $\mathrm{BP}$ in normoxia and the $\mathrm{BP}$ response to acute hypoxia. Our results suggest that increases in $\mathrm{ONOO}^{-}$ in the $\mathrm{CB}$ contribute to the $\mathrm{CB}$ chemosensory potentiation induced by $\mathrm{CIH}$. In addition, $\mathrm{CIH}$-induced systemic hypertension is critically dependent on $\mathrm{ONOO}^{-}$since Ebselen treatment reduces $\mathrm{BP}$ to values similar to the ones measured in normotensive animals. Thus, it is plausible that the primary action of Ebselen reduced the exacerbation of CB chemosensory output and the sympathetic induced hypertension, but we cannot exclude other effects on the hypoxic chemoreflex pathway. To our knowledge, this is the first study that shows that an $\mathrm{ONOO}^{-}$scavenger was effective to prevent the $\mathrm{CB}$ chemosensory potentiation and reverses the hypertension induced by $\mathrm{CIH}$.

A growing body of evidence supports the proposal that the $\mathrm{CB}$ contributes to the autonomic dysfunction and hypertension in OSA patients and animals exposed to CIH. Indeed, patients with recently diagnosed OSA show enhanced ventilatory, pressor, and sympathetic responses to acute hypoxia, attributed to a potentiation of the CB chemoreflexes $[1,2,7]$. Indeed, Narkiewicz et al. [13] found potentiated reflex ventilatory, tachycardic, and pressor responses to acute hypoxia in untreated normotensive patients with OSA. On the contrary, the ventilatory and pressor responses induced by hypercapnia and by the cold pressor test in OSA patients were not different from those observed in control subjects. Similarly, animals exposed to $\mathrm{CIH}$ show enhanced hypoxic ventilatory responses to acute hypoxia [7] for review and long-term facilitation of respiratory motor responses $[8,11]$. Recording 

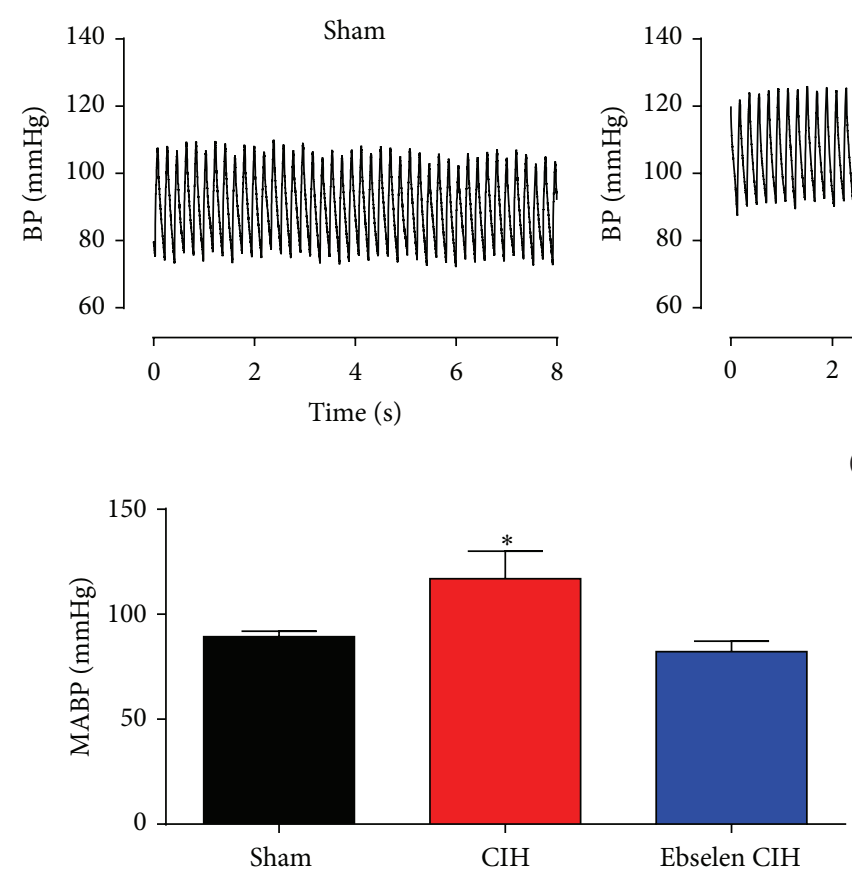

(b)

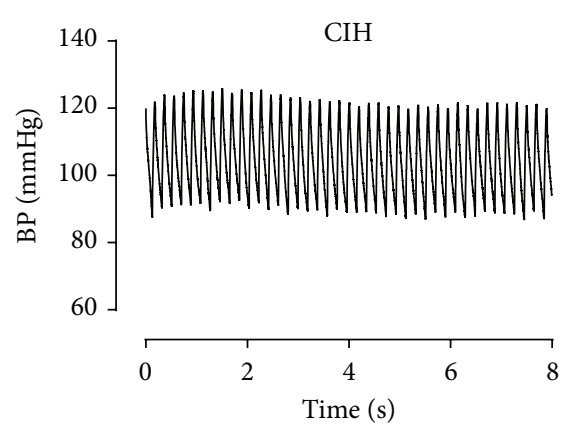

(a)

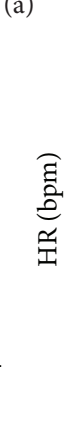

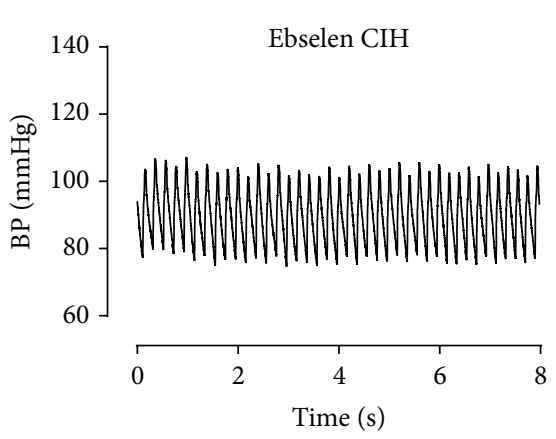

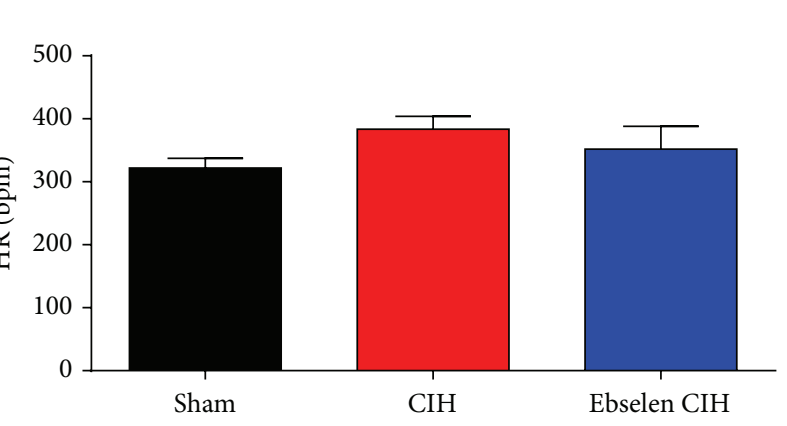

(c)

FIGURE 3: Ebselen reversed the increased arterial blood pressure measured during normoxia in CIH rats. Telemetric recording realized in the same rat during control condition ((a), sham), after 7 days of $\mathrm{CIH}((\mathrm{a}), \mathrm{CIH})$ and the effect of Ebselen after additional 7 days of $\mathrm{CIH}$ ((a), Ebselen CIH). Summary of the effect of Ebselen on mean arterial blood pressure (MABP, (b)) and heart rate $(\mathrm{HR},(\mathrm{c}))$. ${ }^{*} \mathrm{P}<0.05$, CIH versus sham and Ebselen $\mathrm{CIH}$, and Newman-Keuls after Repeated Measures ANOVA, $n=4$ rats.

of chemosensory discharges from the carotid sinus nerve has confirmed the idea that $\mathrm{CIH}$ produces facilitation of the $\mathrm{CB}$ chemosensory responses to hypoxia. Indeed, exposure of rats and cats to $\mathrm{CIH}$ for few days increases the baseline $\mathrm{CB}$ discharges measured in normoxia and enhances the chemosensory responses to acute hypoxia [8-12]. Peng et al. [8] reported that baseline $\mathrm{CB}$ discharge and chemosensory responses to acute hypoxia were higher in rats exposed to short cyclic hypoxic episodes followed by normoxia, applied during 8 hours for 10 days. Similarly, we found that cats and rats exposed to $\mathrm{CIH}$ for 7 days showed enhanced $\mathrm{CB}$ chemosensory and ventilatory responses to acute hypoxia $[8,12]$. Studies performed in OSA patients and animals exposed to $\mathrm{CIH}$ show that OSA is associated with sympathoexcitation, mainly attributed to the enhance $\mathrm{CB}$ chemosensory function elicited by $\mathrm{CIH}[1,2,7]$.

Several studies have proposed that ROS are involved in the progression of the cardiovascular pathologies in patients suffering OSA and animals exposed to $\mathrm{CIH}[2,3,5,7,24]$. Indeed the $\mathrm{O}_{2}{ }^{-}$radical has been proposed as the main ROS responsible for these pathological consequences, since treatment with SOD mimetic prevented the hypertension induced by $\mathrm{CIH}$ in rats $[8,24]$. It is well known that $\mathrm{O}_{2}{ }^{-}$reacts with nitric oxide (NO) producing $\mathrm{ONOO}^{-}$with an elevated constant rate of $\sim 7 \cdot 10^{9} / \mathrm{Ms}$ [25]. Interestingly, this rate is 3.5 times higher than its enzymatic dismutation by SOD [26]. This fast reaction explains how these particularly elusive species could rapidly react to form $\mathrm{ONOO}^{-}$[27], reducing the NO bioavailability $[28,29]$. Accordingly, we previously found a reduction in the NO production in the rat $\mathrm{CB}$ after 7 days of $\mathrm{CIH}$ [30]. Since NO is considered an inhibitory modulator of $\mathrm{CB}$ chemosensory discharges [31], a reduced NO level may partially contribute to enhancing the baseline $\mathrm{CB}$ discharges and chemosensory responses to hypoxia. This interpretation agrees with the observation of Marcus et al. [32], who found that $\mathrm{CIH}$ decreased the expression of the nNOS in the rat $\mathrm{CB}$, suggesting that the removal of the normal inhibitory $\mathrm{NO}$ influence contributes to enhancing the $\mathrm{CB}$ chemosensory responses to hypoxia.

There is evidence suggesting that $\mathrm{ONOO}^{-}$radical is involved in the development of diseases such as type I diabetes, cancer, stroke, heart failure, and neurodegenerative disorders $[33,34]$. The $\mathrm{ONOO}^{-}$radical is highly unstable and produces deleterious reactions and cytotoxic effects, such as oxidation of several molecular targets like lipids, proteins, and DNA [35-37]. One of the main consequences of increased levels of $\mathrm{ONOO}^{-}$is the modification of tyrosine residues in proteins producing 3-NT [27], which has been related with many diseases and cellular damage including liver disease [38], chronic allograft nephropathy [39], and Alzheimer's and Parkinson's disease [40]. We found an increase of 3-NT-ir accumulation in the $\mathrm{CB}$ from rats exposed for 7 to 21 days to $\mathrm{CIH}$, suggesting that $\mathrm{ONOO}^{-}$formation due to the reaction of $\mathrm{NO}$ with $\mathrm{O}_{2}{ }^{-}$is a critical step in the $\mathrm{CB}$ chemosensory potentiation induced by $\mathrm{CIH}[9,18]$. Present results agree with and extend the idea that $\mathrm{ONOO}^{-}$radical contributes to 

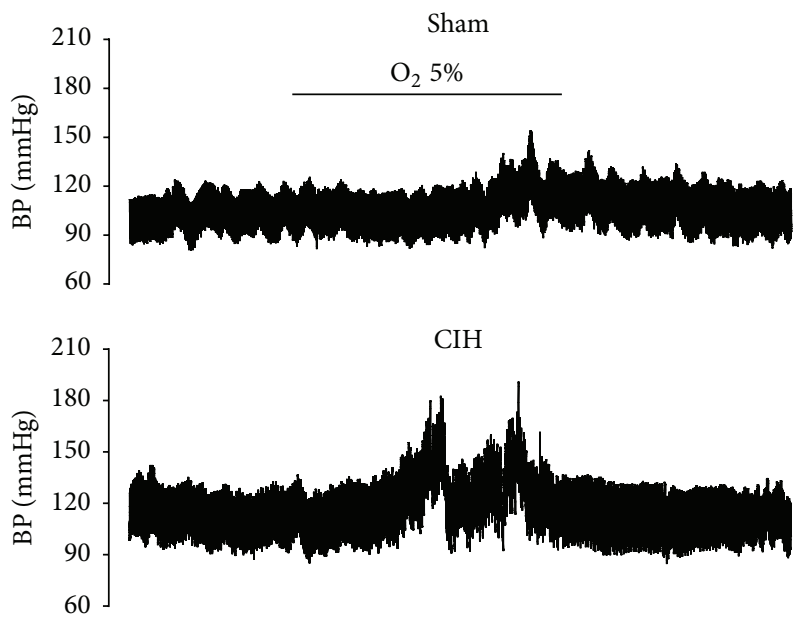

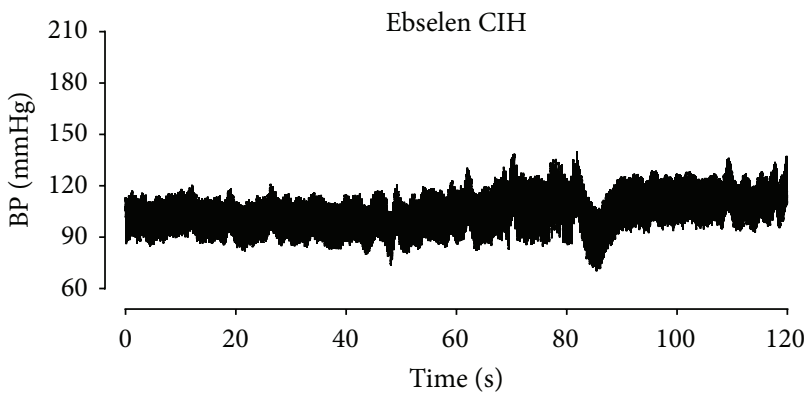

(a)

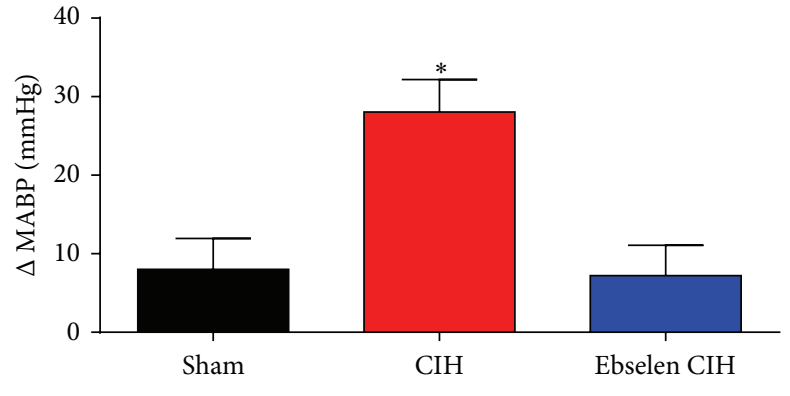

(b)

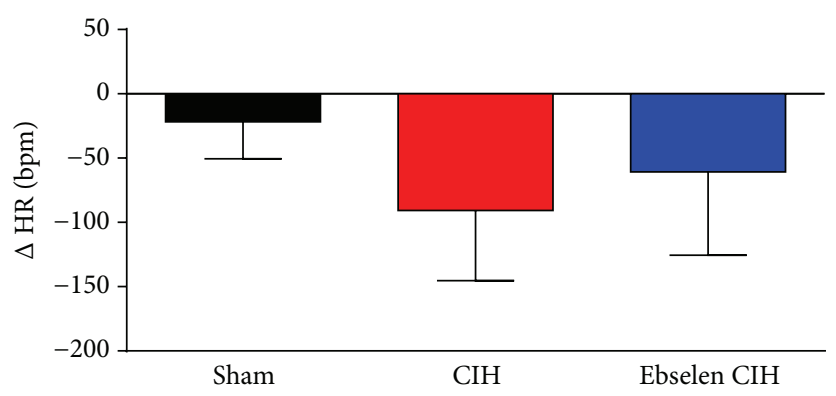

(c)

FIGURE 4: Ebselen reversed the $\mathrm{CIH}$-induced increase in $\mathrm{BP}$ responses evoked by an acute hypoxic stimulus. Telemetry recordings realized in the same rat show the pressure response evoked by hypoxia $\left(5 \% \mathrm{O}_{2}\right.$ for $\left.50 \mathrm{~s}\right)$, during sham conditions, after 7 days of CIH, and after additional 7 days of CIH but supplemented with Ebselen (a). Summary of the effect of Ebselen on mean arterial blood pressure (MABP, (b)) and heart rate $(\mathrm{HR},(\mathrm{c})) .{ }^{*} \mathrm{P}<0.05, \mathrm{CIH}$ versus sham and Ebselen $\mathrm{CIH}$, and Newman-Keuls after Repeated Measures ANOVA, $n=4$ rats.

the $\mathrm{CB}$ enhanced responses to hypoxia after CIH. Ebselen is an organoselenium compound that mimics glutathione peroxidase activity [19-21], which rapidly reacts with $\mathrm{ONOO}^{-}$ [19]. The generation of $3-\mathrm{NT}$ is a direct result of the $\mathrm{ONOO}^{-}$ generation, and the treatment with Ebselen prevented the CIH-induced increase of 3-NT-ir levels in the CB and the chemosensory potentiation of the $\mathrm{CB}$ after $\mathrm{CIH}$, suggesting that protein nitration may play a role in enhancing the chemoreceptor responses to acute hypoxia.

To study plausible therapeutic effects of Ebselen in an experimental model of OSA, we decide to administrate Ebselen after the development of hypertension in rats exposed to $\mathrm{CIH}$. We found that Ebselen treatment effectively normalized resting $\mathrm{BP}$ in awake rats, even in the presence of the intermittent hypoxic stimulus (Figure 3). In addition, Ebselen abolished the potentiated BP response to acute hypoxic stimulation observed during CIH exposure (Figure 4). Taken together, Ebselen administration should be considered as a novel tool to restore normal BP adjustments following $\mathrm{CIH}$. In contrast, we did not find any significant difference between the HR in response to acute hypoxia between the treatments (Figure 4). Acute hypoxia in conscious rats generates a biphasic HR response, characterized by an initial tachycardia during mild hypoxia, but bradycardia when inspired fraction of $\mathrm{O}_{2}$ decrease below $8 \%$ [41]. Then, chronic exposure to intermittent hypoxia may affect both the tachycardic and 
bradycardic responses to acute hypoxia. Future studies are needed to elucidate these questions.

\section{Limitations of the Study}

Our results suggest that Ebselen prevents the nitration of proteins in the $\mathrm{CB}$, which contributes to normalizing the $\mathrm{CB}$ frequency of discharge and the sympathetic-mediated hypertension. Administration of Ebselen was achieved using subcutaneous osmotic minipumps; therefore, the treatment is delivered systemically. Thus, it is possible that Ebselen may act not only in the CB, but also in other parts of the chemosensory pathway (i.e., nucleus of the tractus solitary, rostral ventrolateral medulla), since Ebselen can cross the blood brain barrier [42]. Rats exposed to CIH show increased plasma renin activity [43], and it is known that Losartan treatment prevents the $\mathrm{CIH}$-induced hypertension [43], the increased sympathetic activity induced by apnea episodes [32], and the decrease in arterial vasodilation induced by acetylcholine after 28 days of $\mathrm{CIH}$ [44]. Moreover, intracerebroventricular injection of Losartan prevents the hypertension induced by $\mathrm{CIH}$ and the neuronal activation in areas related to sympathetic activation [45]. Thus, we cannot exclude effects of Ebselen at the central nervous system, sympathetic peripheral system, or arterial vessels, which may all be involved in the antihypertensive effect of Ebselen.

\section{Conclusion}

Present results suggest that 3-NT accumulation contributes to the $\mathrm{CB}$ chemosensory potentiation through the nitration of protein residues, which in turn promotes hypertension. Ebselen treatment prevents the increased CB chemosensory activity and reverses the hypertension in rats exposed to $\mathrm{CIH}$, suggesting that the $\mathrm{CB}$ chemosensory potentiation plays a key role in the generation and maintenance of the hypertension induced by $\mathrm{CIH}$. Further development of $\mathrm{ONOO}^{-}$targeted scavengers should be of therapeutic interest in the treatment of hypertension in OSA patients.

\section{Conflict of Interests}

The authors declare that there is no conflict of interests regarding the publication of this paper.

\section{Authors' Contribution}

Esteban A. Moya, Paulina Arias, Carlos Varela, and María P. Oyarce participated in the experimental studies. Esteban A. Moya and Rodrigo Iturriaga analyzed the data and Esteban A. Moya, Rodrigo Del Rio, and Rodrigo Iturriaga wrote the paper.

\section{Acknowledgments}

This work was supported by Grants FONDECYT 1100405 and 1150040. The authors would like sincerely to thank Dr. Julio Alcayaga for his generous assistance with the design and support necessary to develop the system of intermittent hypoxia used in this study.

\section{References}

[1] V. K. Somers, D. P. White, R. Amin et al., "Sleep apnea and cardiovascular disease an American Heart Association/American College of cardiology foundation scientific statement from the American Heart Association Council for High Blood Pressure Research Professional Education Committee, Council on Clinical Cardiology, Stroke Council, and Council on Cardiovascular Nursing. In collaboration with the National Heart, Lung, and Blood Institute National Center on Sleep Disorders Research (National Institutes of Health)," Circulation, vol. 118, no. 10, pp. 1080-1110, 2008.

[2] J. A. Dempsey, S. C. Veasey, B. J. Morgan, and C. P. O’Donnell, "Pathophysiology of sleep apnea," Physiological Reviews, vol. 90, no. 1, pp. 47-112, 2010.

[3] D. Gozal and L. Kheirandish-Gozal, "Cardiovascular morbidity in obstructive sleep apnea," American Journal of Respiratory and Critical Care Medicine, vol. 177, no. 4, pp. 369-375, 2008.

[4] T. Young, M. Palta, J. Dempsey, J. Skatrud, S. Weber, and S. Badr, "The occurrence of sleep-disordered breathing among middleaged adults," The New England Journal of Medicine, vol. 328, no. 17, pp. 1230-1235, 1993.

[5] L. Lavie, "Obstructive sleep apnoea syndrome-an oxidative stress disorder," Sleep Medicine Reviews, vol. 7, no. 1, pp. 35-51, 2003.

[6] J. F. Garvey, C. T. Taylor, and W. T. McNicholas, "Cardiovascular disease in obstructive sleep apnoea syndrome: the role of intermittent hypoxia and inflammation," European Respiratory Journal, vol. 33, no. 5, pp. 1195-1205, 2009.

[7] R. Iturriaga, E. A. Moya, and R. Del Rio, "Carotid body potentiation induced by intermittent hypoxia: implications for cardiorespiratory changes induced by sleep apnoea," Clinical and Experimental Pharmacology and Physiology, vol. 36, no. 12, pp. 1197-1204, 2009.

[8] Y.-J. Peng, J. L. Overholt, D. Kline, G. K. Kumar, and N. R. Prabhakar, "Induction of sensory long-term facilitation in the carotid body by intermittent hypoxia: implications for recurrent apneas," Proceedings of the National Academy of Sciences of the United States of America, vol. 100, no. 17, pp. 10073-10078, 2003.

[9] R. Del Rio, E. A. Moya, and R. Iturriaga, "Carotid body and cardiorespiratory alterations in intermittent hypoxia: the oxidative link," European Respiratory Journal, vol. 36, no. 1, pp. 143-150, 2010.

[10] R. Del Rio, E. A. Moya, M. J. Parga, C. Madrid, and R. Iturriaga, "Carotid body inflammation and cardiorespiratory alterations in intermittent hypoxia," European Respiratory Journal, vol. 39, no. 6, pp. 1492-1500, 2012.

[11] Y.-J. Peng and N. R. Prabhakar, "Effect of two paradigms of chronic intermittent hypoxia on carotid body sensory activity," Journal of Applied Physiology, vol. 96, no. 3, pp. 1236-1242, 2004.

[12] S. Rey, R. Del Rio, J. Alcayaga, and R. Iturriaga, "Chronic intermittent hypoxia enhances cat chemosensory and ventilatory responses to hypoxia," The Journal of Physiology, vol. 560, no. 2, pp. 577-586, 2004.

[13] K. Narkiewicz, P. J. H. van de Borne, C. A. Pesek, M. E. Dyken, N. Montano, and V. K. Somers, "Selective potentiation of peripheral chemoreflex sensitivity in obstructive sleep apnea," Circulation, vol. 99, no. 9, pp. 1183-1189, 1999. 
[14] S. Rey, M. P. Tarvainen, P. A. Karjalainen, and R. Iturriaga, "Dynamic time-varying analysis of heart rate and blood pressure variability in cats exposed to short-term chronic intermittent hypoxia," American Journal of Physiology-Regulatory, Integrative and Comparative Physiology, vol. 295, no. 1, pp. R28R37, 2008.

[15] R. Iturriaga, D. C. Andrade, and R. Del Rio, "Enhanced carotid body chemosensory activity and the cardiovascular alterations induced by intermittent hypoxia," Frontiers in Physiology, vol. 5, article 468, 2015.

[16] R. Iturriaga, E. A. Moya, and R. Del Rio, "Inflammation and oxidative stress during intermittent hypoxia: the impact on chemoreception," Experimental Physiology, vol. 100, no. 2, pp. 149-155, 2015.

[17] C. Gonzalez, G. Sanz-Alyayate, M. T. Agapito, and A. Obeso, "Effects of reducing agents on glutathione metabolism and the function of carotid body chemoreceptor cells," Biological Chemistry, vol. 385, no. 3-4, pp. 265-274, 2004.

[18] R. Del Rio, E. A. Moya, and R. Iturriaga, "Differential expression of pro-inflammatory cytokines, endothelin-1 and nitric oxide synthases in the rat carotid body exposed to intermittent hypoxia," Brain Research, vol. 1395, pp. 74-85, 2011.

[19] A. Daiber, M.-H. Zou, M. Bachschmid, and V. Ullrich, "Ebselen as a peroxynitrite scavenger in vitro and ex vivo," Biochemical Pharmacology, vol. 59, no. 2, pp. 153-160, 2000.

[20] Y. Nakamura, Q. Feng, T. Kumagai et al., "Ebselen, a glutathione peroxidase mimetic seleno-organic compound, as a multifunctional antioxidant. Implication for inflammation-associated carcinogenesis," The Journal of Biological Chemistry, vol. 277, no. 4, pp. 2687-2694, 2002.

[21] G. K. Azad and R. S. Tomar, "Ebselen, a promising antioxidant drug: mechanisms of action and targets of biological pathways," Molecular Biology Reports, vol. 41, no. 8, pp. 4865-4879, 2014.

[22] J.-G. Park, J.-Y. Yoo, S.-J. Jeong et al., "Peroxiredoxin 2 deficiency exacerbates atherosclerosis in apolipoprotein E-deficient mice," Circulation Research, vol. 109, no. 7, pp. 739-749, 2011.

[23] A. H. Bubolz, Q. Wu, B. T. Larsen, D. D. Gutterman, and Y. Liu, "Ebselen reduces nitration and restores voltage-gated potassium channel function in small coronary arteries of diabetic rats," American Journal of Physiology: Heart and Circulatory Physiology, vol. 293, no. 4, pp. H2231-H2237, 2007.

[24] C. M. Troncoso Brindeiro, A. Q. da Silva, K. J. Allahdadi, V. Youngblood, and N. L. Kanagy, "Reactive oxygen species contribute to sleep apnea-induced hypertension in rats," The American Journal of Physiology-Heart and Circulatory Physiology, vol. 293, no. 5, pp. H2971-H2976, 2007.

[25] M. Valko, D. Leibfritz, J. Moncol, M. T. D. Cronin, M. Mazur, and J. Telser, "Free radicals and antioxidants in normal physiological functions and human disease," International Journal of Biochemistry and Cell Biology, vol. 39, no. 1, pp. 44-84, 2007.

[26] R. A. Dweik, "Nitric oxide, hypoxia, and superoxide: the good, the bad, and the ugly!” Thorax, vol. 60, no. 4, pp. 265-267, 2005.

[27] G. Ferrer-Sueta and R. Radi, "Chemical biology of peroxynitrite: kinetics, diffusion, and radicals," ACS Chemical Biology, vol. 4, no. 3, pp. 161-177, 2009.

[28] A. G. Estévez and J. Jordán, "Nitric oxide and superoxide, a deadly cocktail," Annals of the New York Academy of Sciences, vol. 962, pp. 207-211, 2002.

[29] J. S. Beckman and W. H. Koppenol, "Nitric oxide, superoxide, and peroxynitrite: the good, the bad, and ugly," The American Journal of Physiology -Cell Physiology, vol. 271, no. 5, pp. C1424C1437, 1996.
[30] E. A. Moya, J. Alcayaga, and R. Iturriaga, "NO modulation of carotid body chemoreception in health and disease," Respiratory Physiology \& Neurobiology, vol. 184, no. 2, pp. 158-164, 2012.

[31] R. Iturriaga, M. Mosqueira, and S. Villanueva, "Effects of nitric oxide gas on cat carotid body chemosensory response to hypoxia," Brain Research, vol. 855, no. 2, pp. 282-286, 2000.

[32] N. J. Marcus, Y.-L. Li, C. E. Bird, H. D. Schultz, and B. J. Morgan, "Chronic intermittent hypoxia augments chemoreflex control of sympathetic activity: role of the angiotensin II type 1 receptor," Respiratory Physiology and Neurobiology, vol. 171, no. 1, pp. 3645, 2010.

[33] P. Pacher, J. S. Beckman, and L. Liaudet, "Nitric oxide and peroxynitrite in health and disease," Physiological Reviews, vol. 87, no. 1, pp. 315-424, 2007.

[34] C. Szabó, H. Ischiropoulos, and R. Radi, "Peroxynitrite: biochemistry, pathophysiology and development of therapeutics," Nature Reviews Drug Discovery, vol. 6, no. 8, pp. 662-680, 2007.

[35] F. Yamakura and H. Kawasaki, "Post-translational modifications of superoxide dismutase," Biochimica et Biophysica Acta, vol. 1804, no. 2, pp. 318-325, 2010.

[36] J. T. Hancock, R. Desikan, and S. J. Neill, "Role of reactive oxygen species in cell signalling pathways," Biochemical Society Transactions, vol. 29, no. 2, pp. 345-349, 2001.

[37] J. J. Haddad, "Oxygen sensing and oxidant/redox-related pathways," Biochemical and Biophysical Research Communications, vol. 316, no. 4, pp. 969-977, 2004.

[38] L. A. MacMillan-Crow, D. L. Cruthirds, K. M. Ahki, P. W. Sanders, and J. A. Thompson, "Mitochondrial tyrosine nitration precedes chronic allograft nephropathy," Free Radical Biology and Medicine, vol. 31, no. 12, pp. 1603-1608, 2001.

[39] M. A. Abdelmegeed and B.-J. Song, "Functional roles of protein nitration in acute and chronic liver diseases," Oxidative Medicine and Cellular Longevity, vol. 2014, Article ID 149627, 21 pages, 2014.

[40] A. Martínez, M. Portero-Otin, R. Pamplona, and I. Ferrer, "Protein targets of oxidative damage in human neurodegenerative diseases with abnormal protein aggregates," Brain Pathology, vol. 20, no. 2, pp. 281-297, 2010.

[41] J. P. Casanova, M. Contreras, E. A. Moya, F. Torrealba, and R. Iturriaga, "Effect of insular cortex inactivation on autonomic and behavioral responses to acute hypoxia in conscious rats," Behavioural Brain Research, vol. 253, pp. 60-67, 2013.

[42] N. Singh, A. C. Halliday, J. M. Thomas et al., "A safe lithium mimetic for bipolar disorder," Nature Communications, vol. 4, article 1332, 2013.

[43] E. C. Fletcher, G. Bao, and R. Li, "Renin activity and blood pressure in response to chronic episodic hypoxia," Hypertension, vol. 34, no. 2, pp. 309-314, 1999.

[44] N. J. Marcus, N. R. Philippi, C. E. Bird, Y.-L. Li, H. D. Schultz, and B. J. Morgan, "Effect of AT 1 receptor blockade on intermittent hypoxia-induced endothelial dysfunction," Respiratory Physiology and Neurobiology, vol. 183, no. 2, pp. 67-74, 2012.

[45] W. D. Knight, A. Saxena, B. Shell, T. P. Nedungadi, S. W. Mifflin, and J. T. Cunningham, "Central losartan attenuates increases in arterial pressure and expression of FosB/ $\Delta$ FosB along the autonomic axis associated with chronic intermittent hypoxia," American Journal of Physiology-Regulatory Integrative and Comparative Physiology, vol. 305, no. 9, pp. R1051-R1058, 2013. 


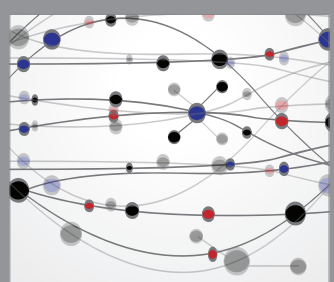

The Scientific World Journal
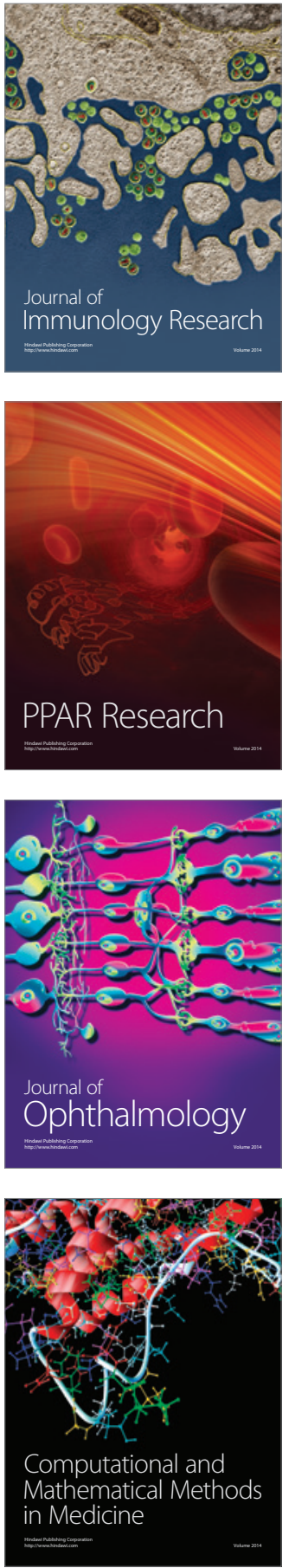

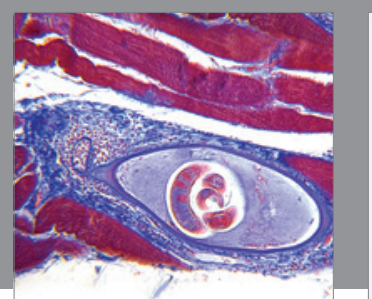

Gastroenterology Research and Practice

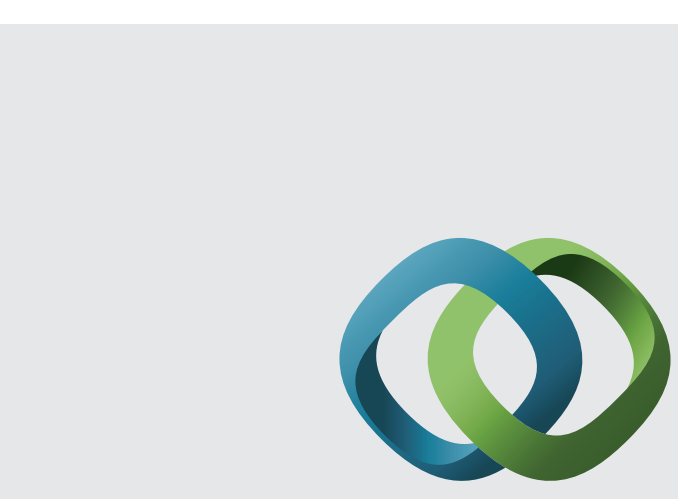

\section{Hindawi}

Submit your manuscripts at

http://www.hindawi.com
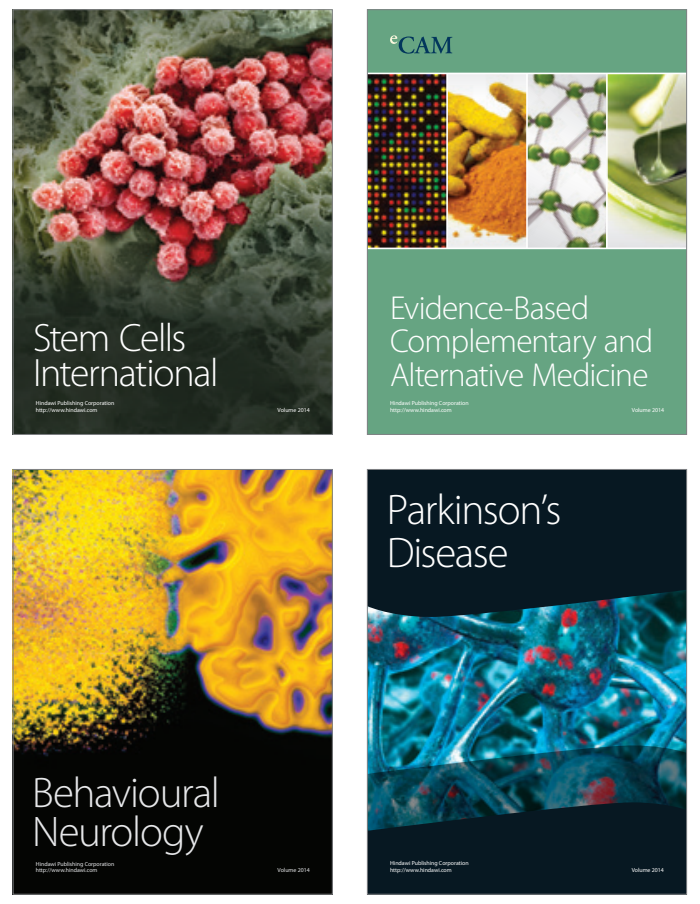
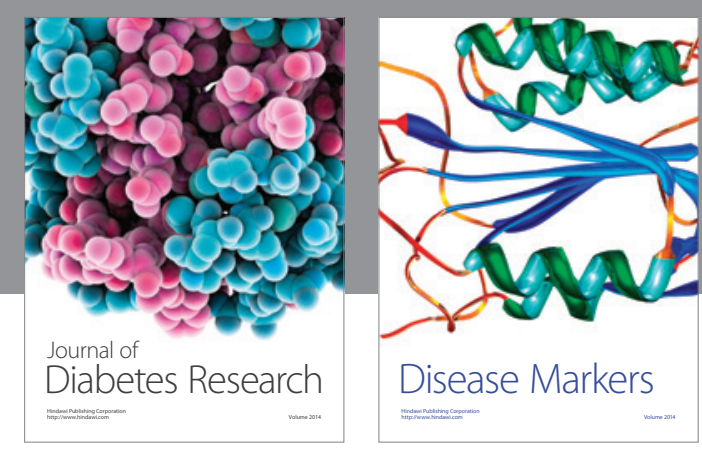

Disease Markers
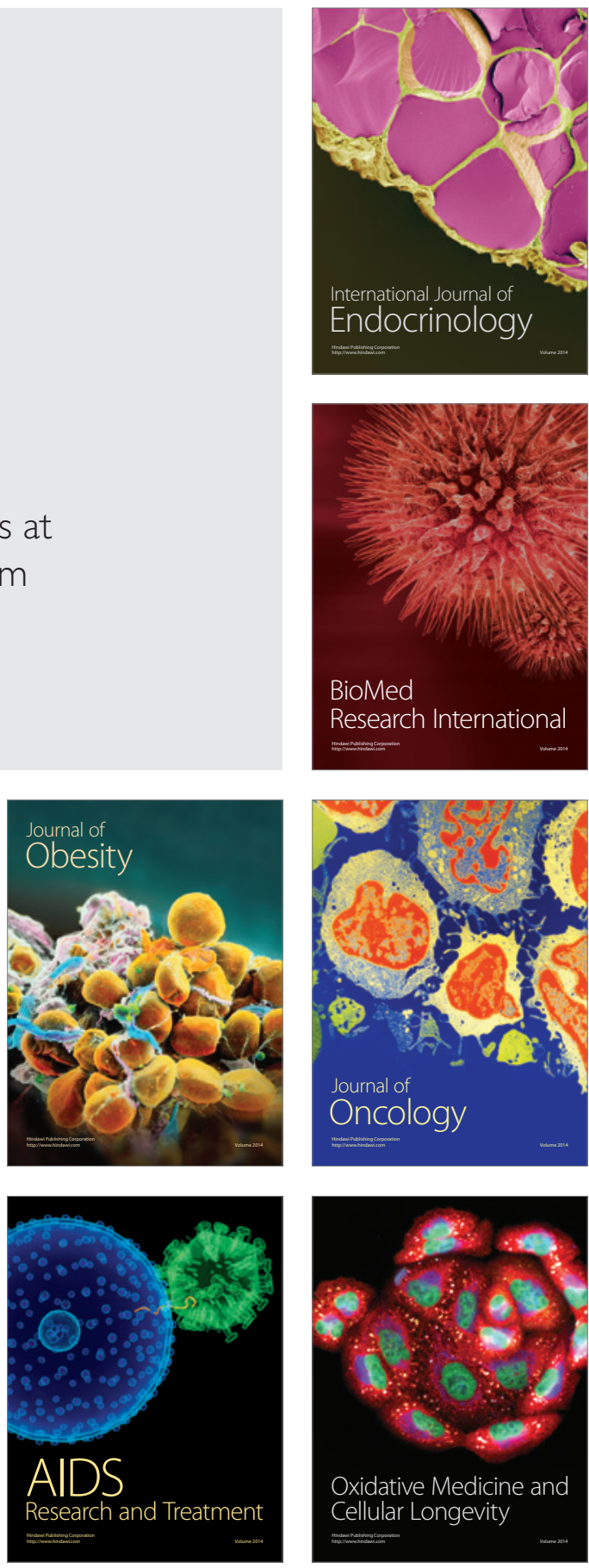